\title{
MULTIPLE STAGE PRODUCTION PLANNING IN PLAIN MANUFACTURING ENVIRONMENTS
}

\author{
GASTERMANN, B[ernd] C[hristian]; LUFTENSTEINER, F[ranz]; \\ STOPPER, M[arkus] \& KATALINIC, B[ranko]
}

\begin{abstract}
In this paper, an implementation case study is presented, which demonstrates the segmentation of the production process in order to achieve highlyflexibile variant management, increased processing throughput and reduced inventory size. At the beginning, fundamentals of each of the relevant concepts and systems are briefly introduced. Then, after some aspects of product classification and variants have been discussed, the case study and its implementation of amultistage manufacturing process areillustrated in detail.Consequently, the interaction of underlyingsoftware processes with specific focus on Infor ERP and a propriety Constant Work-In-Process (CONWIP) planning tool are clarified.
\end{abstract}

Keywords:CONWIP; delivery reliability; inventory downsizing; reduced throughput times; Infor MRP

\section{INTRODUCTION}

A production planning and control (PPC) system is an essential part of production processes of each manufacturing company around the world. Although various manufacturing technologies exist to help with these processes to create products, it is important to make use of only those that are most suitable for the respective production field. Globalization has led to a dramatic change within the competitive environment of the manufacturing ecosystem andas a consequence it became vital to incorporate agile and lean production techniques that increase efficiency, flexibility, quality, and costeffectiveness in order to satisfy quickly changing customer demands and keep up with increasing global competition. Considering that, one approach to accomplish these quality characteristics is discussed by this paper. In form of a case study, the realisation of a multiple stage production process for plain manufacturing environments is presented. The paper briefly introduces the reader to the relevantfundamental concepts and then discusses aspects of the implemented production process with focus on Constant Work-InProcess (CONWIP) and influence on management of variants for plastics industry.

\section{FUNDAMENTALS}

With regard to PPC, a multitude of manufacturing processes exist. However, if broken down into their basic elements and then examined for commonalities, only a few fundamental principles remain.In this section of the paper, some of these basic concepts,from which even complex manufacturing systems are constructed, will be discussed. Although most of these topics here were already covered in-depth by previous papers like [2] and
[3], those that are particularly relevant for this paper are briefly recapped for the sake of completeness.

\subsection{Push and Pull Strategies}

In supply chain management, it is common to classify manufacturing systemsas either push- or pull-based system. Both of them are essential production strategies.

Manufacturing systems are considered push-based, if they release work units according to a master production schedule (MPS). As such, production is triggered by forecast demand and/or historic demand. Actual demand, however, is not being considered. If the release of new work is determined, it is then subsequently pushed through each step of the production line until the work is done and the final product will eventually be stored at the finished goods inventory. This implies that products are always produced in advance, regardless of actual demand. Jodlbauer used to call these products "anonymous", which meansthat they are not associated withanactual consumer[4].Therefore, push systems couldalso be described as "make-to-stock" (MTS).It is self-evident that such abuild-ahead approach heavily relies on accuracy and reliability of demand forecasts.

Contrariwise, pull-based systems do depend on actual demand for the release of new work, though it is irrelevant where it originates from. In other words, the presence of demand, whether it is a customer order or internal demand from other manufacturing stages, pulls raw materials and components through the manufacturing process. Because of this, such systems could also be referred to as "make-to-order" (MTO). With this approach, problems of excessive inventory, which is quite common with the traditional MTS strategy, are relieved so that the amount of products in stock is lower while having more product types available [4]. Generally,pull-based systems allow for more flexibility and product customization.

\subsection{Re-Order Point Planning}

Re-Order Point (ROP) Planning is a method of inventory control used to automate inventory and to minimise total inventory holding costs. ROP itself represents a certain level of stock at which signals to refill the inventoryare triggered. Considering a certain lead time, the ROP must be high enough to allow ordered materials to arrive at the inventory before it is completely empty.The problem of this approach, however, is that replenishment orders are inferred from historic data and demand is expected to not show heavy fluctuations.

\subsection{Constant Work-In-Process}

Reference [7] presented the concept of Constant Work-In-Process (CONWIP) as an enhanced and 
generalized form of Kanban. As the name implies, its basic notion is to ensure a constant level of work-inprocess (WIP) throughout the whole production system. It is, however, neither pure pull nor pure push system, but incorporates aspects of both of them[4].

Although already presented in 1990, CONWIP is still quite unknown and hardly ever used within Europe. A reason for this could be the lack of actual CONWIP implementations forPPC applications.In its most simple form, CONWIP operates as a list-based system where demand triggers the release of new work units, which is a characteristic of pull-based systems. Each of these work unitsis then, similar to Kanban, assigned an global authorization card from asingle global card set, which remains associated to that item until it is complete. Released work unitsarethen pushed through each step of the manufacturing process. As soon as a product is finished, its authorization card is released. Thisallows another work unitto enter the production cycle.Under the assumption that all work units are of equal size, WIP remains constant for the whole production system as the total amount of authorization cards within the CONWIP system is also unchanged. Nontheless, changes in the quantity of cards are allowed. In case ofa bottleneck, for example,cards could be reduced. On the other hand, they could also be increased in order to raise WIP and to ensure higher throughput [5].

As a practical example, it is relatively easy to perform CONWIP-based production planning by means of a production order list [1]. Such a list (as illustrated in figure 1)needs to be filled and maintained by a MPS system that acts independently from the production control system. This list is then sequentially processed by the CONWIP manufacturing system.An interesting aspect of such a list is that it does not require equal lot sizes for all work units. The list is basically divided into four status groups:The topmost section groups completedproduction orders that are already in stock. Typically, this serves informational purpose only. Below is the second groupthat contains items currently in production. The next two groups are for scheduled and pending work units.They appear in either of these two groups, depending on their respective target dates and some other list parameters, which will be discussed now in a few words to provide an overview. For a more detailed disquisition, refer to [3].

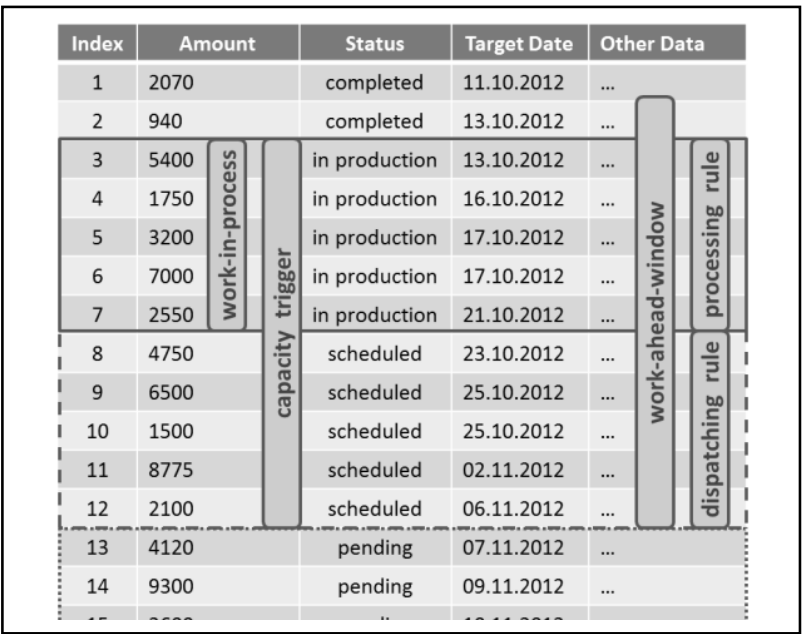

Fig. 1. This illustration shows an example of a CONWIP order list and highlights the scope of its parameters.
With regard to a simple CONWIP production order list, there are various important parameters that influence the arrangement and release of work units[1].

The work-ahead-window (WAW)is the timeframe in which work units are scheduled or released for production according to their respective target dates. The purpose of this parameter is to avoid the release of too much work is in low-selling periods. With this, the system is able to automatically reduce WIP and output quantity to the level of actual demand.

The capacity trigger parameterdefines the maximum amount of work the production line can handle within a certain timeframe without the allocation of additional working resources. Consequently, it helps to detect capacity bottlenecks.

Dispatching and processing rule determine the order in which list items are ordered and processed. The dispatching rule affects the sequential arrangement in which scheduled work units are released. By default, the rule is based on the date of delivery, which causes the item with the earliest delivery date to be released next[7]. However, it is also possible to use different dispatching rules.Similarly, the processing rule determines the order in which released work items are processed by the production line. Although the order of items is defined by these rules, it is still possible to overridethis order manually and prioritize certain work items. However, doing so could have a negative effect on due dates.

At last, aWIP cap limits the maximum amount of work on which the production system is allowed to concurrently work on. It prevents the release of new production orders if the amount of work exceeds the currently set WIP cap. The value for this parameter is usually determined by the bottleneck of the production line.

\subsection{Hybrid Production Strategies}

When designing a manufacturing system, it is not always appropriate to adopt either the MTS or the MTO production approach. However, they are not mutually exclusive [7], so it is possible to combine both of them into a hybrid production system, which is often referred to as "make-to-assemble" (MTA). Hybrid manufacturing strategies are able to combine aspects of both push- and pull-based systems in the following way:

In a first step, basic components of a product are produced and stockedon an intermediate storage based on forecast or pre-defined schedules. As soon as a customer order is placed, the previously stocked components are then used to assemble the final product. With this approach, there is one point that splits the manufacturing chain into MTS and MTO production: The inventory of components clearly marks the point of transition between the two approaches.This point is either called the Order Penetration Point (OPP) [6] or Customer Order Decoupling Point (CODP) [4], though only the latter term will be used throughout this paper. The actual position of this spot may vary, but it is important to wisely choose its location in order to gain benefits from both push and pull strategy. Moving the CODP closer to the customer, for example, improves responsiveness, while moving it farther away from improves flexibility. 


\section{PRODUCTION PLANNING FOR MID-RANGE VOLUME PRODUCTS}

Many companies are facing a hybrid production situation. Customer requirements are increasingly moving towards individual products and so the total product volume spreads across a growing number of product variants. By analysing the product range (for example by means of an ABC analysis), characteristics of MTS, MTO and MTA can be found. Few major products with high volume and a well-known standard design are produced to stock. The customer demand for these products is widely stable and predictable. Companies often refer to these products as A-class products. At the other end of scale we can find C-class products characterised by low volume even down to oneof-a-kind. In an ideal situation the customer accepts longer delivery lead times so that the production orders are based on a customer order. Actually products and components are stocked as well, so that C-class products have a disproportionate share of the total inventories of a company.

In this paper we are focussing on a third category (that of B-class products), which are characterised by a significant share of the total volume and a customerdriven amount of variants. Furthermore the customer demands delivery lead times for these products, which are much shorter than the production lead time. Starting the production with the presence of a customer order, it is not possible to meet customers' needs in terms of delivery lead time. Due to the amount of variants it is also not possible to stock products in every different variant economically. Thus, the MTS approach is not feasible as well.

As discussed above we now have a MTA situation for B-class products in which the CODP divides production into two parts. On the one hand there is the customer orientated stage triggered by actual customer orders. And on the other hand there is a stage where components or parts are produced customer anonymously. Typically the CODP is a stocking point. The production lead time downstream the CODP now is shorter than the delivery lead time. Provided that the right parts are present at the stocking point, customer orders can be delivered in time. Activities upstream the CODP have to ensure the availability of the right parts at the intermediate store.

In terms of PPC we now have two stages of production with different objectives. Downstream the CODP production is customer orientated where the focus is on delivery speed, delivery reliability and flexibility concerning volume and product variants [6]. Not to forget the overall objectives like low price and high quality. The company has to provide flexibility and speed and even be able to respond to demand fluctuations quickly. At the same time inventories have to be low.Upstream the CODP objectives are to maintain high stock availability and to produce at low costs. To meet these different objectives on both sides of the CODP the company has to find an appropriate combination of PPC strategies for every single stage based on an appropriate set of criteria.

In summary, the main criteria for the selection of a PPS strategy are the ability to deal with numerous variants and with demand fluctuation as well as the support of the customer orientated production. Additional criteria are the transparency of the planning process and ease of use.

Material Requirements Planning (MRP) is very popular and has helped many companies reducing inventory levels and improving customer service level in many cases [7]. It is, however, disadvantageous that there are several parameters for every single variant which have to be determined and kept up to date. In opposite to MRP, CONWIP uses only few parameters for a single production line, regardless of the number of variants. In short this means that CONWIP requires less effort for data maintenance.In terms of ease of use and transparency, CONWIP has major advantages compared to MRP. The plain CONWIP list of production orders provides an overview "at a glance" and the planning process follows certain easy-to-understand rules. The results are comprehensible to the production control staff.

The first stage, in which components are produced customer anonymously for the intermediate store, is characterised by high volume und few variants. Additionally fluctuations are much smoother than in the second stage. Based on these assumptions ROP is the PPC strategy for the first stage.

Summarised, the combination of CONWIP and ROP planning is appropriate to address the companies' objectives for the PPC of B-class products.

\section{CASE STUDY: IMPLEMENTING CONWIP}

In this section, focus lies on the case study, in which ROP and CONWIP have been implemented for evaluation in a separated area of a manufacturing plant. The plant is part of a medium-sized manufacturing company operating in the sanitary branch of plastics industry. The motivation of this case study is based on the fact that said company did not incorporate an efficient production planning and control system. Until then, manufacturing planning had been carried out solely by manual methods. This approach may work under certain circumstances, but in order to keep up with competition and increasing demand it became necessary to incorporate an approved and more effective production planning and control system. At first, the requirements for such a system had to be specified. The target was to apply a production system that is transparent, easy to manage, and highly efficient for the required type of production. Classical ERP solutions, for example, provide an extensive range of functionality, but are therefore also mostly afflicted with high complexity. However, high complexity was not a desirable objective, so other production strategies had to be assessed as well. In the end, the decision to make use of a hybrid production strategy has been made because of the inherent advantages of such a manufacturing system.

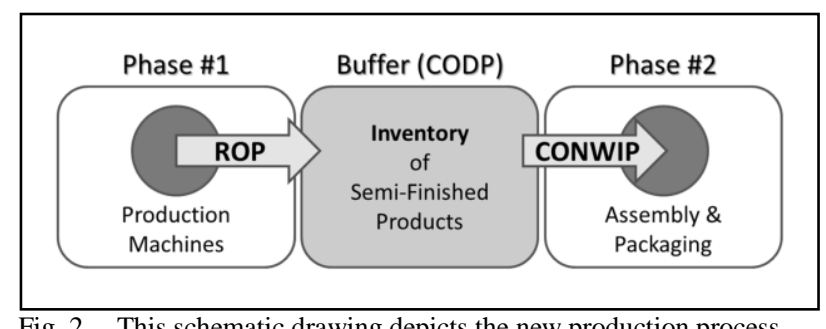

Fig. 2. This schematic drawing depicts the new production process. 
Figure 2 illustrates the layout of the new production process. It is inspired by a typical hybrid production approach that is divided into two manufacturing stages.

For the first stage, a typical MTS production system is used. In this stage, raw materialsare processed by plastic moulding presses, which produce generalized components based on ROP planning. These components are stocked on a buffer, which is an intermediate storage for semi-finished products. The buffer represents the CODP of this whole hybrid system. Using ROP implies that whenever the stock level of the buffer drops below a certain level, production order are automatically created that induce moulding presses to produce the missing parts and refill the buffer. It is generally no problem to realise this stage by means of an existing ERP system, which is required anyway. The Infor ERP, which is used at that manufacturing company, supports this approach.

The second stage is implemented as a MTO system. Here, CONWIP is used for planning and control. As soon as a customer order is placed, an internal assembly and packaging order is created and then scheduled by a CONWIP planning tool, which might just be an order list as described in the previous section. For each released order, components are taken from the buffer and a specific variant of the product is constructed. Eventually, the final products are packaged and sent to the customer. Unlike in the first phase, assembly and packaging is initiated only by actual demand, that is, customer orders.

However, difficulties arise from the fact that CONWIP is not a well-known approach to work with. As already mentioned, it was no problem to use the existing Infor ERP system, for ROP-based planning. But as expected, CONWIP was not supported. Because of this, the decision to develop proprietaryplanning softwarewas made. Details on this software are planned to be published in a subsequent paper.

\section{PROCESSES OF PLANNING SOFTWARE}

As seen above, the presence of new production orders represents the starting point in the CONWIP planning process. Basically, customer orders are captured and converted to production orders in the existing ERP system and transferred to the CONWIP software. According to its desired delivery date, every single production order appears in the CONWIP list together with relevant data like amount, required capacity, and availability of materials.

On a weekly basis sums of capacity utilised by the current planning situation are calculated. In order to take planning decicions, these calculated capacity sums are then contrasted with currently provided capacity. If necessary, production orders are allowedto be moved within the WAW. Once the delivery date of an order fulfills relevant criteria, it is acknowledged and its changedtarget date is transferred back to the ERP system to allow for a notification to the customer.

With CONWIP, production orders should be released as late as possible. On one hand, work stations must not run out of work, provided that production orders are present within the WAW. On the other hand, very late production orders have to be taken into account, which provides a maximum of flexibility.
Status changes, like orders that are released for production,are transferred to the ERP system as well. All further steps until completion of the production order are conducted within the ERP system. Finally, the status change of completed orders is transferred to the CONWIP software again, which moves the respective list item to the area of completed production orders.

\section{CONCLUSION}

Companies having a product range with variants are facing an ambitious production situation. Depending on product volume and the number of available variants, characteristics of MTS, MTO and MTA can be found. In this paper it is pointed out that different PPC approaches have to be implemented for every different area to bring together customer requirements and the companies' objectives.

Based on an $\mathrm{ABC}$-analysis it is shown that there is a group of B-class products, which consists of numerous product variants and represents a significant share of the total product volume. The position of the CODP yields a MTA situation. In the customer orientated part of the production, flexibility and speed are essential. Additionally, demand fluctuations are significant. In the customer anonymous part of production the most important point is to reduce costs. Furthermore, ease of use and transparency are considered as an important criterion for selecting a PPC system.

The presented combination of CONWIP and ROP is just one approach on how manufacturing companies could achieve ambitious objectives for B-class products. It is able to reduce WIP, but also able to increase flexibility and speed and to deliver numerous product variants demanded by the customer.

\section{REFERENCES}

[1] Altendorfer, K. \& Jodlbauer, H. (2007). CONWIP - Hohe Liefertreue bei gleichzeitig niedrigen Beständen, In: PPS Management, Vol. 2007, No. 1, Gronau, N., (Ed.), pp. 16-19, Gito, ISBN 9783940019011 , Berlin

[2] Gastermann, B. C.; Stopper, M. \& Katalinic, B. (2011). Aspects of the Production Control System CONWIP in Small and Medium Sized Industrial Companies, Annals of DAAAM for 2011 \& Proceedings of the 22nd International DAAAM Symposium, Katalinic, B., (Ed.), pp. 621-622, ISBN 9783901509834, ISSN 1726-9679, DAAAM International, Vienna, Austria, 2011

[3] Gastermann, B. C. \& Stopper, M. (2012).Conceptual Prototype of a Planning Software for the CONWIP Production Control System, Proceedings of the International MultiConference of Engineers and Computer Scientists 2012, Vol. 2, (March 2012), pp. 13341339, ISBN 9789881925190, ISSN 2078-0958, International Association of Engineers, Hong Kong, China, 2012

[4] Jodlbauer, H. (2008). Produktionsoptimierung: Wertschaffende sowie kundenorientierte Planung und Steuerung, Springer, ISBN 9783211781401, Vienna

[5] Marek, R. P.; Elkins, D. A. \& Smith, D. R. (2001). Understanding the Fundamentals of Kanban and CONWIP Pull Systems using Simulation, Proceedings of the 2001 Winter Simulation Conference, Peters, B.A., (Ed.), pp. 921-929, ISBN 9780780373075, Arlington, IEEE Computer Society, Washington

[6] Olhager, J. (2003). Strategic Positioning of the Order Penetration Point. International Journal of Production Economics, Vol. 85, No. 3, (September 2003) pp. 319-329, ISSN 0925-5273

[7] Spearman, M. L.; Woodruff, D. L. \& Hopp, W. J. (1990). CONWIP: a Pull Alternative to Kanban. International Journal of Production Research, Vol. 28, No. 5, (May 1990) pp. 879-894, ISSN 0020-7543 